\title{
DETECTION OF EXPLOSIVE MIXTURES IN THE ULLAGE OF AIRCRAFT FUEL TANKS
}

\author{
Shin-Juh Chen ${ }^{1}$ and Joel A. Silver ${ }^{2}$ \\ Southwest Sciences, Inc. \\ 1570 Pacheco Street, Suite E-11 \\ Santa Fe, New Mexico 87505
}

\begin{abstract}
Sensors for detecting explosive mixtures in the ullage of aircraft fuel tanks along with inerting systems are of paramount importance for preventing fires and explosions during ground and flight operations of both commercial and military aircraft. A diode laser-based oxygen sensing system is designed and tested to assess its ruggedness and performance in simulated flight conditions and fuel tank environment. This sensor system incorporates recent innovations in microelectronics such as digital signal processors, new vertical surface cavity lasers, high sensitivity absorption spectroscopy, and mechanisms for preventing liquid interferences on optical surfaces. Metal foams and sintered metals of different porosities were tested and found to prevent liquid interferences as a result of liquid sloshing/splashing and immersion without compromising the sensor's time response. Liquid droplets on optical surfaces can interfere with the sensor's performance. Gravity-driven flow on surfaces can remove most of the liquid. Kerosene fuel tends to form a thin liquid film on optical surfaces. The sensor's performance was not adversely affected by this thin film. However, coatings and motions encountered during flight could further enhance the removal of liquid off optical surfaces.
\end{abstract}

\section{INTRODUCTION}

$\underline{\text { Motivation }}$

The effective prevention and control of fires and explosions originating in fuel systems during in-flight, maintenance, and post-crash are critical for both commercial and military aircraft. From 1970 to 1993, thirteen military aircraft suffered total hull loss as a result of non-combat fuel tank explosions [1]. From 1959 to 2001, fourteen commercial aircraft have experienced fuel tank explosions [1] which resulted in 231 fatalities worldwide. As the liquid fuel is consumed, a space (also called ullage) containing air and fuel vapors is developed above the liquid fuel. This fuel-air mixture is potentially dangerous if an explosive composition, favorable environmental factors, and an ignition source are present. Methods to reduce the oxygen concentration in this fuel-air mixture are necessary for the survivability of both commercial and military aircraft. These methods displace the oxygen inside the ullage with

1. AIAA Member; Senior Research Scientist

2. Principal Research Scientist

Copyright $(2004$ by Southwest Sciences, Inc.

Published by the American Institute of Aeronautics and Astronautics, Inc. with permission. nitrogen. Moreover, monitoring systems are also needed to accurately determine the oxygen content inside the ullage and the quality of the inerting gas.

\section{Onboard Inert Gas Generation System}

Replacing the fuel vapor-laden space (ullage) inside the fuel tank as the liquid fuel is being consumed with NitrogenEnriched Air (NEA) minimizes fires and explosions due to potential ignition sources such as lightning strikes, artillery shells, static discharge, wiring sparks, and heating sources. Air separation technologies for the Air Separation Module (ASM) include pressure swing adsorption, hollow fiber membrane, ceramic membrane, cryogenic air separation, and hybrids [2]. The NEA contains usually more than 90 percent nitrogen. The ullage oxygen concentration should be between 9-12 percent to eliminate potential in-tank fires and explosions [2] due to all possible ignition sources.

An Onboard Inert Gas Generation System (OBIGGS) generally encompasses an ASM to generate NEA, a compressor, storage tanks, and a distribution system. The military has OBIGGS currently installed in the AH-64, C-5, $\mathrm{C}-17, \mathrm{~F}-22$, and V-22 to reduce oxygen levels below the

American Institute of Aeronautics and Astronautics 
lower explosive limit inside the fuel tanks. OBIGGS is currently being considered for commercial aircraft to reduce the likelihood of in-tank fires and explosions. Ground and flight tests are already underway to design effective inerting systems for the commercial aircraft [3-5].

\section{The Need for Oxygen Sensors}

Oxygen sensors for monitoring the NEA exiting the Air Separation Module (ASM) of OBIGGS and inside the ullage are required to fully assess the effectiveness of inerting systems. Currently, there is no commercial oxygen sensor system that has been flight-certified to operate in the ullage of aircraft fuel tank. Zirconium oxide oxygen sensors are used to monitor the quality of the NEA at the ASM outlet for military aircraft. However, these sensors cannot be used in commercial aircraft since the sensor operates at high temperature and can be an ignition hazard.

Having the sensor inside the fuel tank provides the best and most direct approach to assess fire and explosion hazards. Existing technologies based on electrochemistry (e.g. zirconium oxide), fluorescence quenching, and paramagnetic properties of oxygen for measuring oxygen concentrations are not suitable for aircraft fuel tanks because the presence of fuel vapor in the ullage prevents safe sensor operation and inhibits optimal performance. Diode laser-based sensor systems may be the only viable solution for monitoring oxygen inside aircraft fuel tanks.

\section{Objective of this Research}

This paper will focus on the design of a diode-laser based oxygen sensor system that is compatible with the aircraft fuel tank environment and flight conditions. This sensor system integrates recent technological advancements in microelectronics such as digital signal processors, new vertical cavity surface emitting lasers, high sensitivity absorption spectroscopy, and the development of innovative shields to prevent liquid interferences of optical surfaces. In particular, methods for minimizing the optical surfaces from getting wet during sloshing/splashing and immersion scenarios are investigated.

\section{METHODS}

$\underline{\text { Absorption Spectroscopy }}$

The measurement of chemical species concentrations by diode laser absorption, as depicted in Fig. 1, is based on straightforward implementation of Beer's law,

$$
\frac{I}{I_{0}}=\exp (-\alpha), \quad \alpha=S(T) g(v) n \ell=\sigma(v) n \ell
$$

where $I_{0}$ is the light intensity incident on the gas sample, $I$ is the transmitted intensity and $\alpha$ the absorption coefficient. The absorption coefficient is comprised of the absorption line strength $S(T)$, the line shape function $g(v)$, number density $n$ in $\mathrm{cm}^{-3}$ and path length $\ell$ in $\mathrm{cm}$. The line strength is a measure of how strongly light is absorbed and is a function of temperature but not pressure. The line shape function describes the wavelength dependence $(v)$ of the absorption and does depend on both temperature and pressure. The absorption cross section term $\sigma(v)$ is the product of $S(T)$ and $g(v)$, and has units of $\mathrm{cm}^{2}$. For conditions of varying temperatures, the line shape function is best described by a Voigt profile [6].

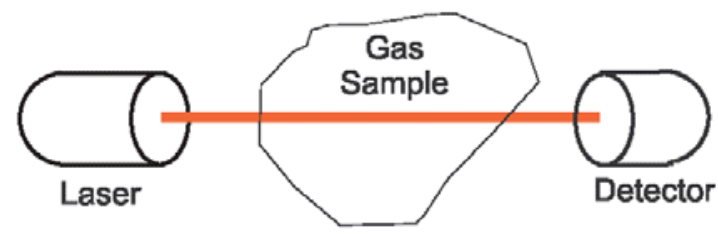

Figure 1. Optical absorption schematic

In the low absorbance limit $(\alpha<<1)$,

$$
\left(I_{o}-I\right) / I_{o}=\alpha
$$

and the fractional absorbance is linear in concentration and quantitative. This method is a line-of-sight technique, so that the absorbances are always spatial integrals along the optical path. Since the quantity actually measured is the ratio of $I$ to $I_{0}$, the resulting concentration is independent of any laser intensity fluctuations and depends only on accurately-known parameters. The low absorbance limit is valid here.

\section{Wavelength Modulation Spectroscopy}

High-sensitivity [7-10] is achieved by using wavelength modulation spectroscopy (WMS) to shift the detection band from DC (where laser amplitude noise can obscure weak absorbances) to high frequencies where laser excess (1/f) noise is unimportant. Fractional absorption sensitivities 
near the shot-noise limit $\left(\sim 10^{-7}\right)$ can be achieved [9]. Field measurements using WMS routinely maintain minimum detection absorbances of better than $10^{-5}$ (at $1 \mathrm{~Hz}$ bandwidth) for extended (many month) operations [11-13]. For comparison, direct transmission methods such as FTIR are only capable of detecting $10^{-3}$ absorbances.

To implement WMS, a small sinusoidal modulation at frequency $f$ is superimposed on the diode laser injection current. This current modulation produces a modulation of the laser wavelength, since wavelength is tuned by changing the current. The amplitude of the current modulation is chosen so that the induced wavelength modulation is comparable to the width of the spectral feature under study. Absorption by the target gas converts the laser wavelength modulation to an amplitude modulation that induces ac components in the detector photo-current. Phase-sensitive electronics are then used to demodulate the detector photo-current at a selected harmonic, $n f$ (typically, $n=2$ ). Studies have shown that detection frequencies as low as $20 \mathrm{kHz}$ are often sufficient to bypass laser excess noise.

The WMS signal is directly proportional to the absorbance,

$$
\text { Signal }=C I_{o} \alpha(v)
$$

where $C$ is a system calibration factor and can be accurately predicted from known electronics factors and spectroscopic properties of the system. Since the cross section, path length and $I_{0}$ are readily known or measured, the gas concentration is determined directly.

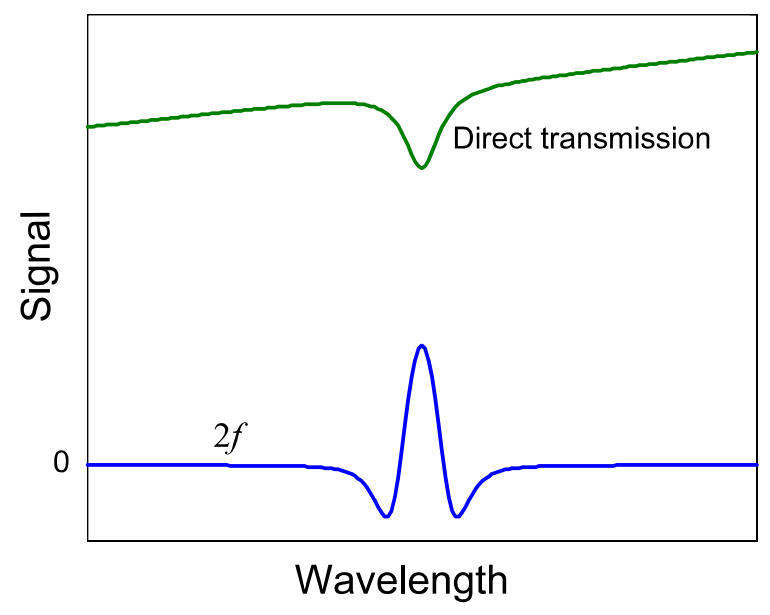

Figure 2. Direct transmission and corresponding $2 f$ signals.
The signal from this detection method appears as approximately the second derivative of the absorption feature with respect to wavelength, as shown in Fig. 2. To first order, diode lasers have linear current versus intensity curves. Thus, $2 f$ signals have a zero background.

\section{Oxygen Sensor System}

The oxygen sensor system is composed an in situ probe for measuring oxygen concentrations in the ullage of an aircraft fuel tank, a pre-amplifier board for signal processing, a DSP system for control, data acquisition and processing on-thefly, and a computer for data visualization and storage. A schematic of the system is shown in Fig. 3.

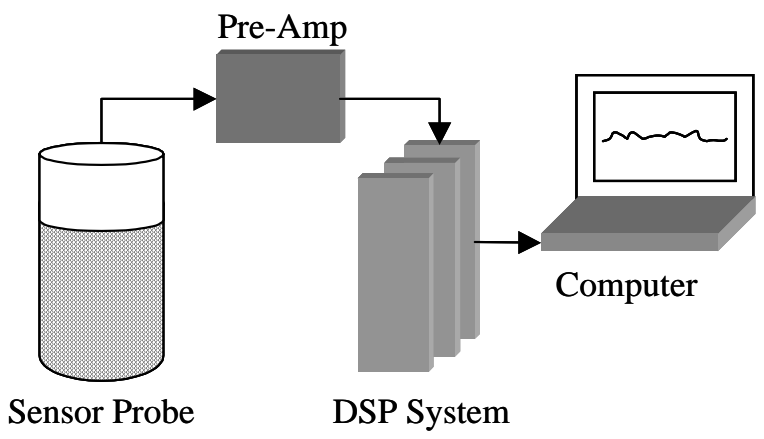

Figure 3. Schematic of the oxygen sensor system showing the sensor probe with metal foam, pre-amp, DSP system and a computer.

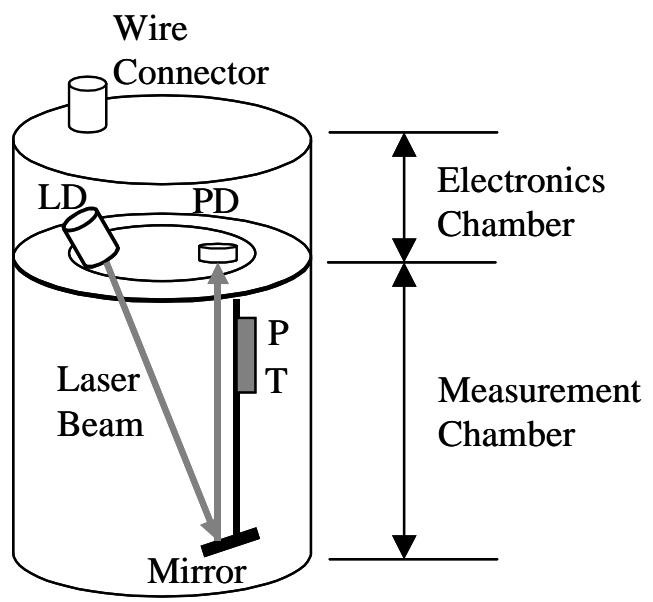

Figure 4. Schematic of the sensor probe showing the electronics and measurement chambers. Laser diode (LD) and photodiode detector (PD) are in the electronics chamber. Pressure (P) and temperature (T) sensor, and mirror are in the measurement chamber. 
The sensor probe is made of two chambers as shown in Fig. 4. The top chamber is about 3.5 inches long and 3.5 inches in diameter. This chamber houses a vertical cavity surface emitting laser which provides the light source for the wavelength modulation spectroscopy, and a silicon photodiode detector for collecting the transmitted intensity. On the bottom of this section a quartz window provides optical access into the lower chamber, while protecting the electronics from the ambient environment. A focusing lens is attached to the laser and the whole system sits on an optical mount which allows both coarse and fine positioning of the laser beam into the bottom chamber. This top chamber can be sealed from the environment and filled with an inert gas such as nitrogen during operation inside the aircraft fuel tank. A pressure sensor can be also installed inside to monitor the integrity of this sealed chamber.

The lower chamber is the measurement region where a mirror lies near the bottom to reflect the laser beam back into the top chamber onto the photodiode detector. The laser beam is reflected off the mirror surface only once to generate a total optical path length of about $20 \mathrm{~cm}$. Pressure and temperature sensors are installed inside this chamber to monitor the ambient conditions and for quantifying the oxygen measurements. The optical surfaces inside this chamber needs to be protected from liquid splashing/sloshing and immersion without comprising the sensor's time response.

\section{Vertical Cavity Lasers}

A recent innovation in diode lasers is the development of vertical cavity surface emitting lasers (VCSEL). In contrast to conventional distributed feedback (DFB) single-mode lasers, which emit from the side of a complex semiconductor chip requiring many structural layers and fabrication steps, VCSELs emit upwards from the surface and as a result are easier to produce, can be tested during fabrication and can be more densely packed, thus lowering costs. Presently, these lasers are commercially available at wavelengths between 750 and $2050 \mathrm{~nm}$ [14]. Research is rapidly pushing the range of wavelengths towards both the UV (shorter wavelengths) and near-infrared (longer wavelengths).

VCSELs are extremely efficient and require only between 3 and $10 \mathrm{~mA}$ to operate. They can tune over $10-50 \mathrm{~cm}^{-1}$ of wavelength, have collimated circular beams, and have output powers near $0.5 \mathrm{~mW}$, which is more than sufficient for wavelength modulation spectroscopy. The low voltage and low laser power make this VCSEL-based oxygen sensor system intrinsically safe. For comparison, conventional DFB lasers (generally available from 1000 to $2000 \mathrm{~nm}$ ) require 10-20 times more power, have highlydiverging, astigmatic beams, and most importantly, only tune (via injection current) over about a single wavenumber. A consequence of the wide tunability of VCSELs is that selection of the optimal absorption line and/or detection of multiple gases is possible using a single laser. Size, optical and power requirements are significantly reduced as well. These VCSELs have been demonstrated to operate in environmental temperatures ranging from -60 to $60{ }^{\circ} \mathrm{C}$, and at pressures as low as 20 Torr [15].

\section{Control and Data Acquisition and Processing}

The DSP system is comprised of three piggy-backed 2" $\times 6$ " circuit boards - a laser controller board, the DSP board and an I/O board. The laser controller board is used to provide needed circuitry beyond the standard features of a generalized DSP system. It contains only the thermoelectric cooler (TEC) control for the laser operating temperature, line drivers/receivers for all I/O devices, inputs for flow and temperature health monitors, and a set of LEDs for diagnostic purposes.

The I/O board contains two high-speed (570 kHz, 18-bit) analog-to-digital (A/D) converters and four slower ones (200 kHz, 16-bit). It also has six digital-to-analog output converters $(200 \mathrm{kHz}, 16$-bit). All of these are tied through a 100-pin interface connector to the laser controller board, using active line drivers and receivers to assure minimal noise and distortion. The high speed ADCs have a six-pole, $100 \mathrm{kHz}$ anti-aliasing filter to assure that the WMS inputs are clean. Thirty-two digital I/O lines are also available. One D/A is used to generate the wavelength ramp and modulation waveform to each laser. The high speed A/D digitizes the WMS signal for subsequent analysis. The slower A/D's is used to read ambient temperature and pressure, and other required control or health monitoring parameters.

The DSP control board includes a Texas Instruments TMS320C6711 processor, an FPGA (flash programmable gate array), $16 \mathrm{MB}$ of SDRAM for data storage and $2 \mathrm{MB}$ of Flash ROM to store the program. The DSP processor stores and runs a C-based software program, which is written on a regular PC and tested using a JTAG interface to the DSP board. Once the program is fully debugged, it is compiled, linked and converted to a binary file which is 
then burned into flash ROM memory on the DSP board.

In addition to this three board DSP package, an additional board is used. This small circuit board plugs into the electrical pins from the laser and photodiode, and from this board a shielded coaxial cable runs to the main electronics unit. This circuit board contains a pre-amplifier for the photodiode, a photoconductive bias circuit (to permit larger input signals while maintaining better linearity), connections to housing and ambient temperature sensors, and the VCSEL laser current driver. This last device is mounted as close to the laser as possible because of the very low electrical currents used to drive this laser.

This system operates using a wall plug $6 \mathrm{~V}$ transformer or four-AA $1.5 \mathrm{~V}$ batteries. Output communications provided are an RS-232 (or RS-488) serial communications port, with optional capabilities (requiring straightforward
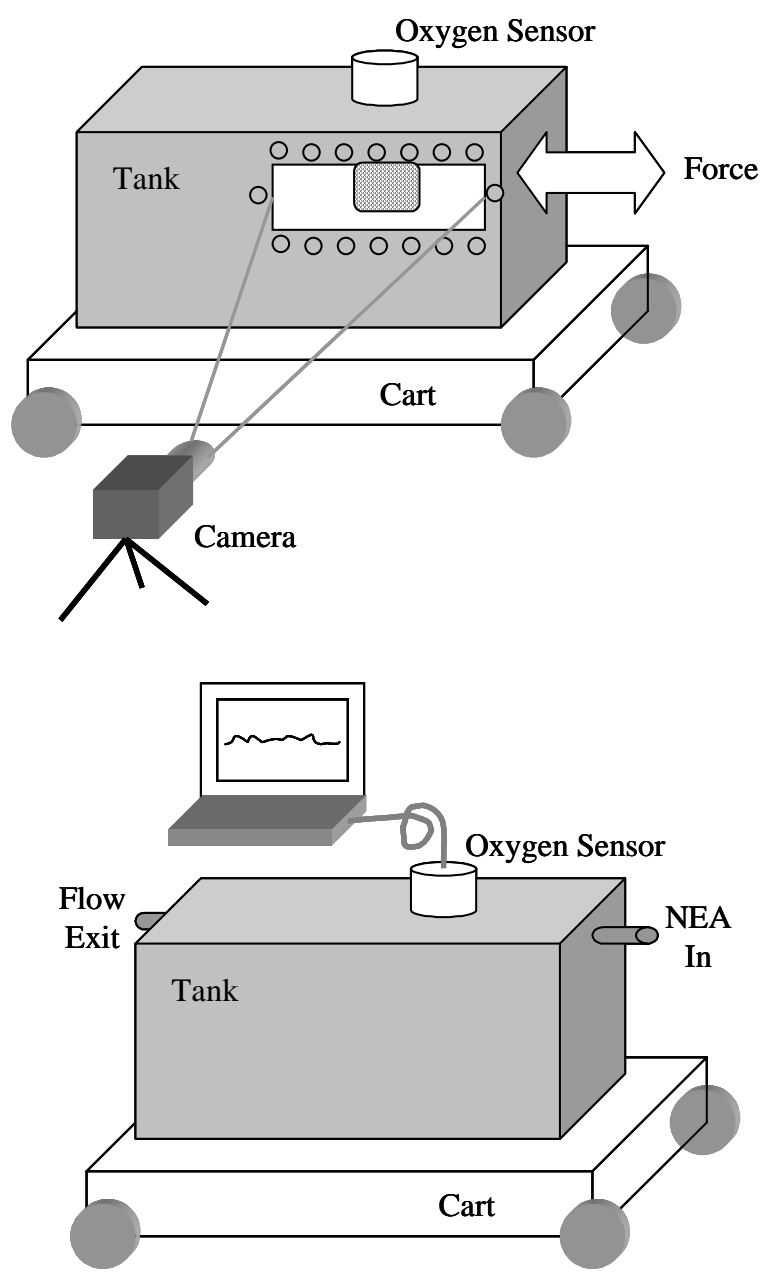

Figure 5. Schematic of the simulated fuel tank for sloshing/splashing tests (upper) and inerting tests (lower). modifications) for a USB port, or (wireless) modem. The entire DSP/control package is extremely compact. It weighs about 6 ounces, has an overall size of $6 \frac{1 / 4}{}$ " $\times 2 \frac{1}{2}$ " $\times$ $1 \frac{1}{2}$ ", and uses only a few Watts of electrical power.

Upon powering the system, the DSP board automatically loads and executes the program. The laser is brought to the set point temperature, then turned on and data acquisition begins. Multiple high speed spectral scans are co-averaged and the WMS data are demodulated, normalized, and fit to theoretical reference spectra to determine the local concentration of gas. The results of these fits, fitting statistics, and sensor health parameters are reported out via the communications port once per second (or as frequently as desired). The system could also be modified to use separate reference gases for analysis.

\section{Simulated Liquid Sloshing/Splashing and Immersion}

A 27 gallon rectangular plastic container was modified for the liquid sloshing/splashing tests (see Fig. 5). The tank is 12.5 inches wide, 30.75 inches long, 19.75 inches high, and has a wall thickness of 0.25 inch. A rectangular section of 18 inches wide and 5 inches high is cut out in one side and replaced by a transparent plexiglass for camera access. A 4.35 inch diameter hole is made on the top of the tank for mounting the sensor probe which extends about 5 inches into the interior of the tank. Metal foams of different porosities are used to assess their efficacy in minimizing or preventing the wetting of optical surfaces in the event of liquid sloshing and splashing. The tank is set on a fourwheel cart and sliding motions are applied to generate waves inside the tank for simulating in-tank sloshing and splashing.

Sintered metal discs of different porosities (6 to 35 micron average pore size) are mounted to one end of a cylindrical hollow tube with the other end closed. The discs are 2 inches in diameter and with thicknesses ranging from 1/16 to $1 / 4$ inch. The end having the disc is immersed in liquid (water or kerosene) for several hours and monitored for leakage of liquid into the cylinder. These sintered metals are useful for designing a measurement chamber that is impermeable to liquids but allow gases to pass through the chamber.

\section{$\underline{\text { Simulated Fuel Tank Inerting }}$}

A second rectangular plastic container is used for simulating fuel tank inerting (see Fig. 5). The sensor probe 


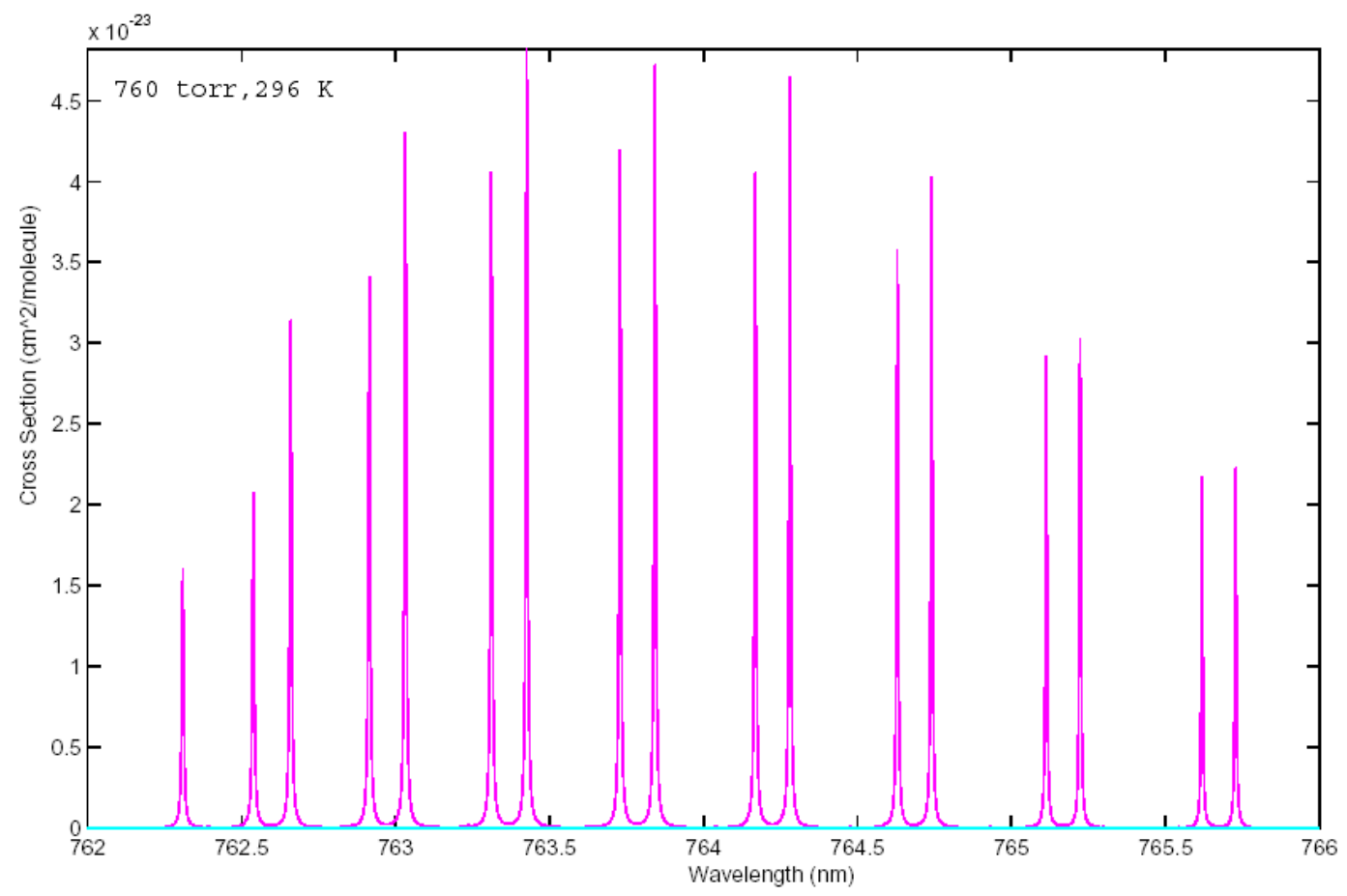

Figure 6. Oxygen absorption spectrum.

is mounted into the center top section of the tank. Two $3 / 8$ inch holes are drilled on opposite ends near the top of the tank for the inflow of Nitrogen-Enriched Air (NEA) and outflow of gas. A certified mixture of 5\% oxygen and $95 \%$ nitrogen is fed into the tank at a flow rate of $0.1 \mathrm{gal} / \mathrm{sec}$. Flow rate is metered using a flow metering valve attached to a flow controller and computer. The effect of metal foams and sintered metals on the time response of the sensor system is determined.

\section{$\underline{\text { Simulated Wetting of Optical Surfaces }}$}

A hand pump is used to generate droplets of water and kerosene. Liquid droplets are sprayed unto the reflecting mirror located in the lower chamber of the sensor probe, and the sensor's response to the liquid droplets is monitored. The liquid sprays range from fine $(1 / 4 \mathrm{~mm}$ diameter) and dispersed spray to the flooding of the optical surfaces. The experiments were all conducted in ambient air and provided insight on additional mechanisms needed to remove liquid off optical surfaces.

\section{RESULTS AND DISCUSSION}

$\underline{\text { Sensitivity and Accuracy of Sensor System }}$
Using HITRAN [16], Fig. 6 shows the oxygen absorption band in the $762-766 \mathrm{~nm}$ spectral region which has well resolved lines. This spectral region is generally inactive and is free of interference from other ambient and fuel species. One important advantage of an optical detection approach is that fuel vapor does not introduce background absorbance signals. This occurs for two reasons. First, hydrocarbons do not have significant absorption bands in the near-visible spectral region $(\sim 760 \mathrm{~nm})$. Second, the very weak bands that do occur are spectrally broad and diffuse, whereas the WMS methods are sensitive only to sharp spectral features such as the lines in Fig. 6.

The effects of altitude (using Standard Atmosphere) on the sensitivity of the laser-based system is shown in Fig. 7. As altitude increases, the absorbance per total optical path length (in $\mathrm{cm}$ ) and oxygen concentrations (in percent) is decreased. From equation 1, the absorbance is linearly proportional to the total optical path length and the number density. At 35,000 feet, using a total optical path length of $100 \mathrm{~cm}, 10$ percent oxygen gives an absorbance of 0.01 with an achievable accuracy of \pm 0.1 percent. Clearly, for most of the commercial aircraft, the change in altitude will have a minimal effect on the sensitivity and accuracy of a diode laser-based oxygen sensor system.

The ambient oxygen concentration was monitored over 


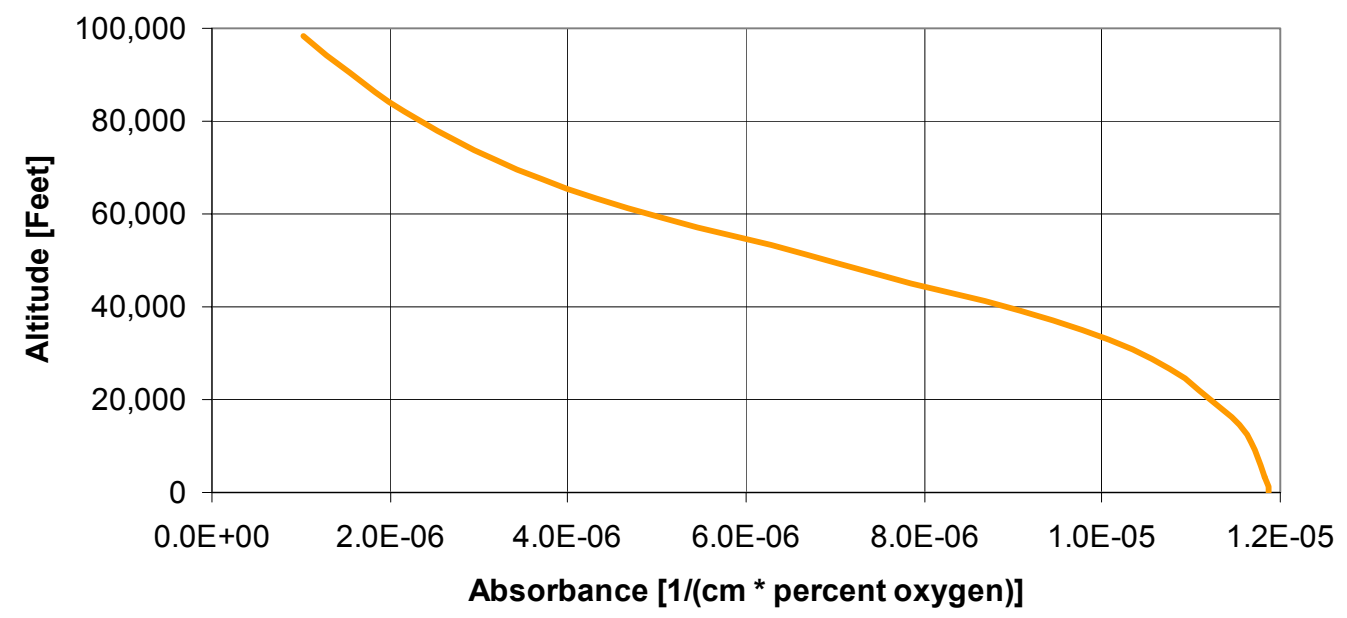

Figure 7. Effects of altitude on the sensitivity of the diode laser-based oxygen sensor system using Standard Atmosphere.

4300 seconds to determine the stability of the system. A standard deviation of 0.2 percent was found for the sensor system. Figure 8 shows a plot of the ambient oxygen concentration over the monitoring period. Averaging over one minute gives a standard deviation of about 0.3 percent. Note that the total optical path length was only $20 \mathrm{~cm}$. Certainly increasing the total optical path length will increase the precision of the measurements. A Herriott cell [17] can be used to generate very large optical path lengths (order of meters) with a very short and low-volume measurement chamber.

\section{$\underline{\text { Slosh/Splashing Guard }}$}

During a typical operation of an aircraft, unsteady motions (due to air turbulence, sudden acceleration and de-

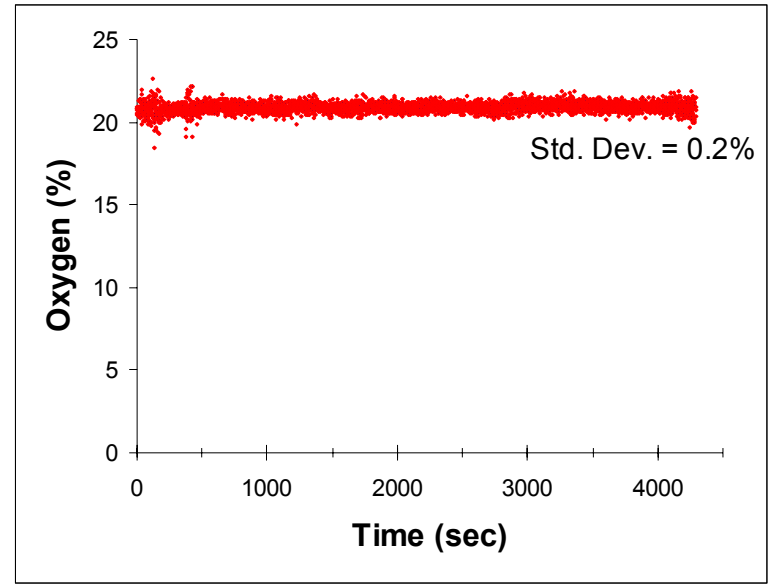

Figure 8. Stability of oxygen sensor system at ambient conditions. Each dot represents data at each second. acceleration) can modify the structure of the liquid layer inside the fuel tank. Even if the sensor probe was flush with top wall of the fuel tank, scenarios of liquid splashing onto the sensor probe is still possible. The liquid layer can move back and forth, filling up certain sections of the tank while emptying others. Sloshing motions with various amplitude and frequency can develop. Preventing the optical surfaces from getting wet is important to minimize the degradation of the sensor's performance. However, any preventive measure must not considerably increase the time response of the sensor to the changes in oxygen concentration inside the fuel tank (such as during inerting).

A metal foam shaped like a tall cup is used to enclose the lower chamber of the sensor probe. A metal bracket holds the cup to the upper chamber. The cup is highly porous and is 5 inches tall, 3.25 inches in diameter and 0.25 inches thick. The reflecting mirror for the optical path is located off-center near the bottom of the cup. The rectangular tank designed for liquid tests is half-filled with water and secured to a four-wheel cart. The tank is rocked back and forth to generate sloshing patterns, and the measurement chamber was splashed several times. Liquid easily drains off the metal foam. After a few sloshing tests, the sensor probe is removed from the tank, and the metal foam is removed to reveal the reflecting mirror. In all sloshing tests, the mirror never got wet. As water splashes onto the metal foam, the metal foam absorbs the impact energy. The liquid droplets do not penetrate the cup's wall to reach the interior. The liquid fills the pores inside the metal foam, and drains off quickly at the bottom of the cup. Preventing the wetting of optical surfaces due to sloshing/splashing can 


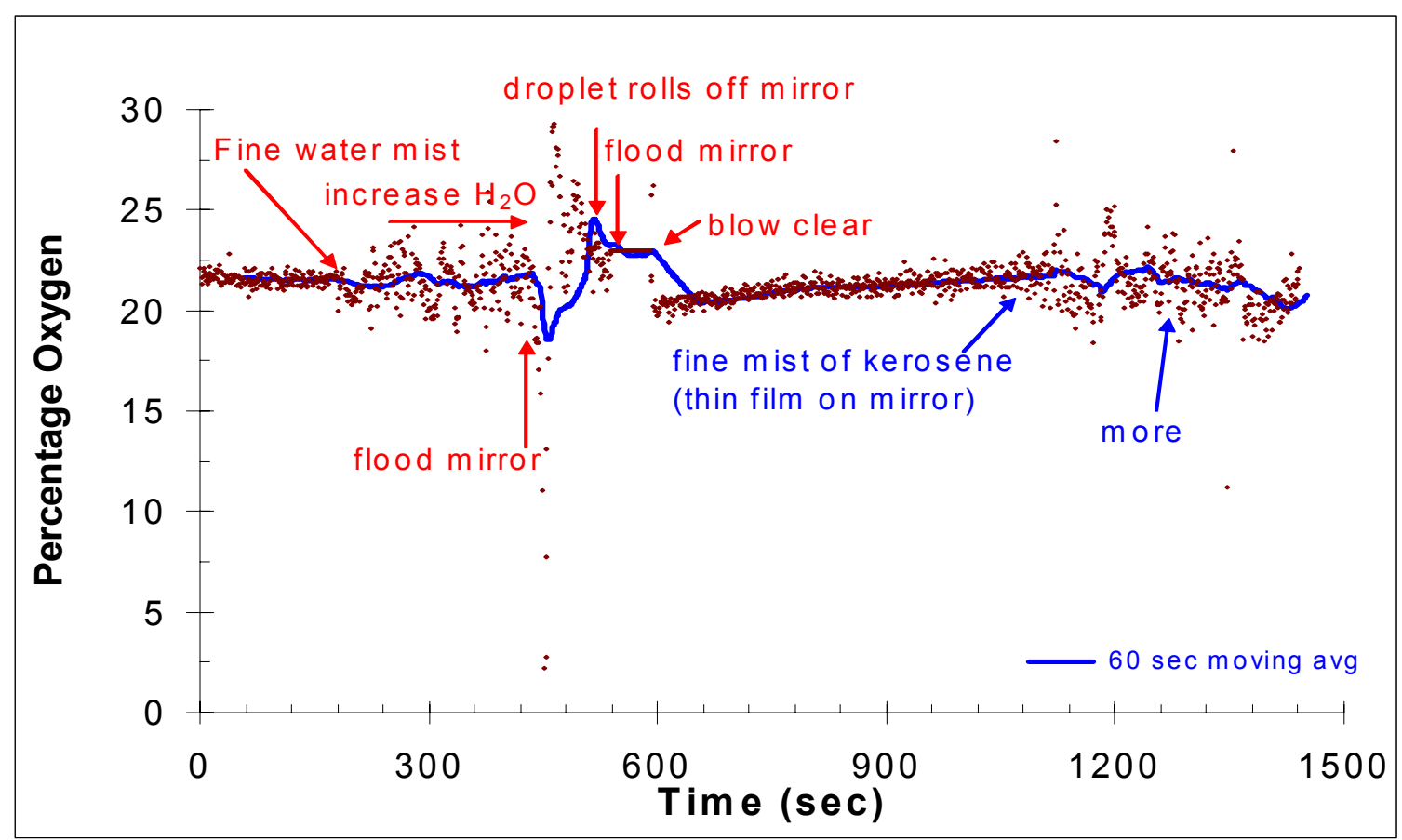

Figure 9. Water and kerosene droplets on optical surfaces and their effects on the sensor's performance.

be accomplished using a metal foam design which encloses the measurement chamber while allowing the chamber to breathe.

\section{$\underline{\text { Liquid Immersion of Sintered Metal Discs }}$}

Sintered metals discs with average pore size ranging from 6 to 35 microns were immersed in liquids (water and kerosene) to assess their impermeability to liquids. In all cases of liquid immersion (up to several hours), liquid was not found inside the testing chamber. Selection of the appropriate pore size depends on the required sensor's time response to changes in oxygen concentrations and the disc's impermeability to liquid.

\section{Wetting of Optical Surfaces}

The reflecting mirror inside the measurement chamber is angled about 15 degrees from the horizontal. This was necessary to redirect the incoming laser beam onto a photodiode detector located inside the upper chamber. Any liquid deposited on the surface will initially runoff due to gravity and leave a thin liquid film due to surface tension. The competition between surface tension and gravity will affect how fast the liquid film runs off the surface. Sprays of water and kerosene were deposited on the mirror surface, and the sensor's performance was monitored.
Figure 9 shows the sensor's response to various sprays of water and kerosene. Fine water droplets $(<1 / 4 \mathrm{~mm})$ on the mirror surface do not degrade the sensor's performance as seen in times between 150 and 450 seconds. Although the data collected appeared scattered, the 60-second moving average shows a reasonable performance. Since water droplets will bead on the mirror surface, the increase in water droplets lead to the formation of larger droplets via coalescence as the droplets roll down the surface due to gravity. Once the droplet diameter is larger than the laser beam diameter (about $1 / 2 \mathrm{~mm}$ ), the laser beam can no longer reflect off the mirror surface and reach the photodiode detector. This can be seen in times between 450 and 600 seconds in Fig. 9. Blowing the surface to remove the water droplets recovers the sensor's normal operation.

As for the kerosene droplets, the increase from a fine spray to the point of flooding does not lead to the loss of laser signals. The kerosene droplets do not bead on the mirror, but forms a thin liquid film. Although the one second data looked scattered, the 60 -second moving average still shows a reasonable concentration of oxygen.

The vibrations and accelerations encountered during normal flight conditions can further enhance the removal of liquid droplets or liquid film off the optical surface. Obviously, a 


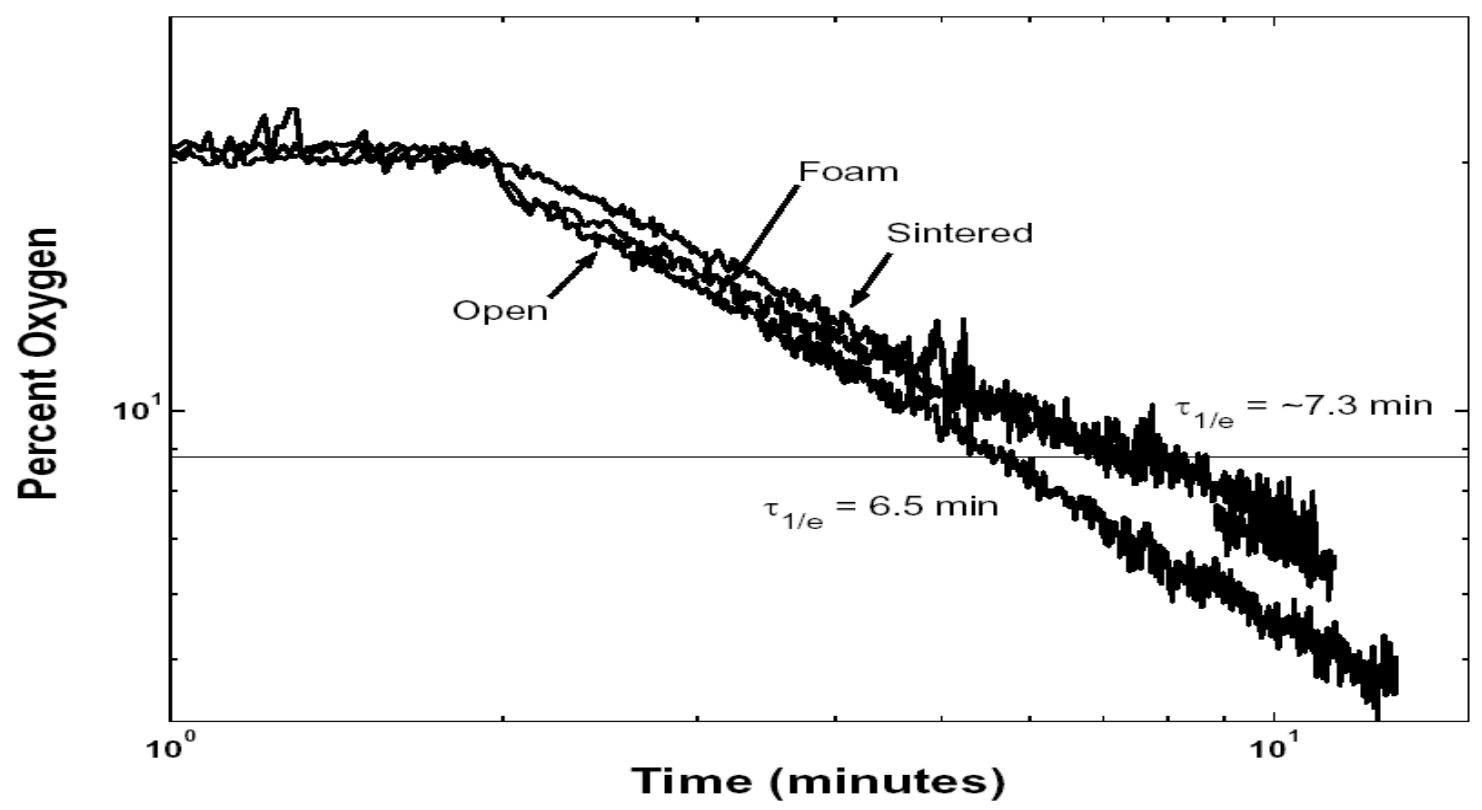

Figure 10. Time response of sensor system with the measurement chamber open, surrounded by a metal foam cup, and closed with a metal tube having a sintered metal disc at the bottom.

coating that is compatible with the fuel environment can further improve the removal of liquid off optical surfaces.

\section{$\underline{\text { Simulated Fuel Tank Inerting }}$}

Using the 27 gallon rectangular tank designed for the inerting tests, the lower chamber of the sensor probe was subjected to three test conditions to estimate the relative diffusion rates of air into the chamber. These are (1) open chamber, (2) chamber surrounded by a metal foam, and (3) a metal tube with a sintered metal installed at the bottom. Oxygen concentrations were recorded each second. For each test condition, the tank started with an ambient oxygen concentration. NEA is fed to the tank at a flow rate of 0.1 gallon per second.

Figure 10 shows the oxygen concentration as a function of time for the three test conditions. Data were collected until the oxygen concentration inside the tank is below 8 percent. To reach a 1/e concentration (i.e 7.7 percent $\mathrm{O}_{2}$ ), it took 6 minutes for the open chamber to reach this level. However, it took only one additional minute for the metal foam and sintered metal test conditions to reach 1/e oxygen point. As expected, the sensor probe with a metal foam has a time response between that of a sensor with an open chamber and sintered metal disc. The results suggest that using a metal foam and sintered metal to minimize the wetting of optical surfaces due to sloshing/splashing and immersion scenarios do not considerably increase the time response of the sensor system. A time response of one minute should be sufficient for monitoring oxygen concentration in the ullage and quality of NEA at the ASM outlet. Under real operating conditions, the aircraft motion of the liquid should reduce the mixing times. For oxygen measurements below 10 percent, a longer total optical path length would be required to reduce the noise seen in Fig. 10.

\section{SUMMARY AND CONCLUSIONS}

To prevent fires and explosions originating from aircraft fuel tanks, the ullage must be inerted using nitrogenenriched air. Oxygen sensors are needed to monitor the ullage and quality of nitrogen-enriched air. A diode laserbased oxygen sensor system was determined to be a feasible sensor for probing the ullage of aircraft fuel tanks. The laser-based system is immune to fuel vapors and environmental conditions encountered during flight.

For a laser-based system to be successful, the optical surfaces must be kept free of liquid droplets. Gravitydriven flow on optical surfaces can remove most of the liquid. A thin liquid film is typically formed when kerosene fuel contact the optical surfaces. Such thin liquid film did not adversely affect the performance of the sensor system. Mirror coatings and motions encountered in flight can 
further aid in the removal of liquid off the optical surfaces.

Mechanisms for preventing or minimizing the entry of liquid into the measurement chamber can include metal foams and sintered metal parts. These mechanisms were shown to provide sufficient protection from liquid sloshing/splashing and immersion without sacrificing the sensor's time response.

\section{ACKNOWLEDGMENTS}

This work was supported by the National Science Foundation under the Small Businesses Innovative Research (SBIR) program with grant no. 0319786.

\section{REFERENCES}

1. Federal Register, Department of Transportation, Fuel Tank Ignition Prevention Measures, Vol. 62, No. 64, Thursday, April 3, 1997.

2. Reynolds, T. L., Eklund, T. I. and Haack, G. A., Onboard Inert Gas Generation System/Onboard Oxygen Gas Generation System (OBIGGS/OBOGS) Study, Part II: Gas Separation Technology - State of the Art, NASA CR-2001-210950 (2001).

3. Burns, M. and Cavage, W. M., Inerting a Vented Aircraft Fuel Tank Test Article with NitrogenEnriched Air, DOT/FAA/AR-01/6 (2001).

4. Burns, M. and Cavage, W. M., Ground and Flight Testing of a Boeing 737 Center Wing Fuel Tank Inerted with Nitrogen-Enriched Air, DOT/FAA/AR01/63 (2001).

5. Burns, M. and Cavage, W. M., A Description and Analysis of the FAA Onboard Oxygen Analysis System, DOT/FAA/AR-TN03/52 (2003).

6. Hui, A. K. Amstrong, B. H., and Wray, A. A., Rapid Computation of the Voigt and Complex Error Functions, J. Quant. Spectrosc. Radiat. Transfer 19, 509-516 (1978).

7. Silver, J. A., Frequency Modulation Spectroscopy for Trace Species Detection: Theory and Comparison Among Experimental Methods, J Appl. Opt. 31, 707717 (1992).

8. Silver, J. A. and Stanton, A. C., Two-Tone Optical Heterodyne Spectroscopy Using Buried Double Heterostructure Lead-Salt Diode Lasers, Appl. Opt. 27, 4438 (1988).

9. Bomse, D. S., Stanton, A. C., and Silver, J. A.,
Frequency Modulation Spectroscopy For Trace Species Detection: Experimental Comparison of Methods, Appl. Opt. 31, 718-731 (1992).

10. Bomse, D. S., Dual-Modulation Laser Line-Locking Scheme, Appl. Opt. 30, 2922 (1991).

11. Stanton, A. C., Silver, J. A., Bomse, D. S., Oh, D. B., Hovde, D. C., Paige, M. E., and Kane, D. J., Applications of Diode Laser Spectroscopy to Environmental and Industrial Process Monitoring, in Application of Tunable Diode and Other Infrared Sources for Atmospheric Studies and Industrial Process Monitoring, A. Fried, ed., Proc. SPIE 2834, 41 (1996).

12. Silver, J. A. and Hovde, D. C., "Near-Infrared Diode Laser Airborne Hygrometer," Rev. Sci. Instrum. 65, 1691-1694 (1994).

13. Hovde, D. C., Meyers, T. P., Stanton, A. C. and Matt, D. R., "Methane Emissions from a Landfill Measured by Eddy Correlation Using a Fast Response Diode Laser Sensor," J. Atmos. Chem. 20, 141 (1995).

14. Vertilas, www.vertilas.com

15. Paige, M. E., Diode Laser Water Vapor Sensor for Weather Balloons, R02-01, Southwest Sciences Internal Report (2001).

16. L.S. Rothman, R.R. Gamache, et al., J. Quant. Spectrosc. Radiat. Transfer 48:469 (2001).

17. D. Herriott, H. Kogelnik and R. Kompfner, "OffAxis Paths in Spherical Mirror Interferometers," Appl. Opt. 3, 523-526 (1964). 